\title{
PRODUCTIVIDAD DE Eucalyptus globulus EN LA REGIÓN DEL MAULE
}

\author{
Vallejos, Oscar $^{1}$ y Tobar, Julio ${ }^{2}$
}

\section{RESUMEN}

Se comparó la productividad de plantaciones de Eucalyptus globulus en 436 parcelas establecidas en la Región del Maule. La comparación se llevó a cabo mediante un análisis de varianza del incremento medio en volumen por hectárea (IMA del volumen). Se consideró como fuente de variación la serie de suelo que sustentaban las plantaciones y como covariable el número de árboles por hectárea, ya que este no permaneció constante.

El análisis de varianza indicó la existencia de diferencias significativas entre la fuente de variación, conformándose tres grupos homogéneos. Como no se cumplieron los supuestos de este análisis, se recurrió a la prueba no paramétrica de Kruskal Wallis, la cual ratificó las diferencias del análisis paramétrico. Los suelos Graníticos conforman el grupo con el menor IMA en volumen (7,6 $\mathrm{m}^{3} /$ ha/año). El grupo intermedio está conformado por los suelos Aluvial Mixto, Metamórficos y Sedimentos Marinos (9,3; 10,7 y 10,8 $\mathrm{m}^{3} /$ ha/año, respectivamente). El grupo destacado está formado por las Cenizas Volcánicas y los Rojo Arcillosos (16,5 y 17,0 m 3 /ha/año, respectivamente).

Los programas de forestación con esta especie deberían considerar los resultados obtenidos en esta investigación a fin de cumplir con las expectativas de los propietarios.

Palabras clave: Eucalyptus globulus, Rendimiento plantaciones, Suelos forestales, Región del Maule.

\footnotetext{
${ }_{2}^{1}$ Facultad de Ciencias Forestales, Universidad de Talca, Chile. ovallejo@utalca.cl

${ }^{2}$ Forestal MININCO S.A. Julio.Tobar@forestal.cmpc.cl
} 


\section{SUMMARY}

Eucalypts planted forest productivity in 436 plots established in the Maule region was compared. The comparison was carried out through a variance analysis on the medium volume annual growth (MAG). As variation source soils series were considered and as covariable the tree number by hectare since this remains not constant.

The variance analysis indicates significant differences between the variation source showing three homogeneous groups. Since the analysis assumptions were not accomplished, the Kruskal Wallis nonparametric test was developed and this test confirmed the parametric analysis differences. Granitic soils form the minor volume MAG group $\left(7.6 \mathrm{~m}^{3} / \mathrm{ha} /\right.$ year $)$. The intermediate group is represented by Mixed Alluvial, Metamorphic and Marine Sediments soils (9.3; 10.7 and $10.8 \mathrm{~m}^{3} /$ ha/year, respectively). The better group included the Volcanic Ashes and the Red Clay Soils (16.5 y $17.0 \mathrm{~m}^{3} / \mathrm{ha} /$ year, respectively).

Eucalypt afforestation programs in the region should consider these results in order to meet the owner's expectations.

Key Word: Eucalyptus globulus, Planted forest yields, Forest soils, Maule Region. 


\section{INTRODUCCIÓN}

El Censo Agropecuario y Forestal del año 2007 indicó la existencia de 22.146 ha de plantaciones de Eucalyptus globulus en la Región del Maule (INE, s.f.), lo que equivale al 5,8\% de la superficie forestada a nivel nacional con la especie. La Región se ubica en cuarto lugar en términos de superficies forestadas con esta especie.

Pampolina et al. (2002) indican que esta especie es la más ampliamente plantada en las regiones de clima mediterráneo, debido a que se adapta a una amplia gama de climas y suelos, posee buenas aptitudes para la fabricación de papel de calidad y principalmente a su rápido crecimiento.

Una de las primeras recopilaciones sobre el incremento volumétrico en la Región del Maule la hizo el Instituto Forestal en la década de los 80 (INFOR-CORFO, 1989). Con posterioridad, algunas publicaciones aportaron antecedentes de ensayos en áreas específicas de la Región (Santelices, 2005; Guerra et al 2010), pero no hay referencias actualizadas a nivel regional, de modo que el presente estudio pretende aportar en este ámbito utilizando una amplia muestra dispersa en toda la Región.

\section{OBJETIVOS}

Maule.

Determinar la productividad de las plantaciones de Eucalyptus globulus en la región del

\section{MATERIAL Y MÉTODO}

En la Región del Maule se establecieron 436 parcelas circulares de $400 \mathrm{~m}^{2}$ cada una, en las tres zonas de crecimiento predefinidas en la Región (Figura $N^{\circ} 1$ ).).

En cada parcela se midieron todos los DAP con forcípula de brazos paralelos graduada al milímetro y la altura de 10 árboles con un clinómetro Suunto graduado cada 0,5 m.

De todos los árboles muestreados fueron seleccionados 175 a los cuales se les hizo análisis fustal, con el objetivo de definir funciones de ahusamiento y de volumen.

En cada zona se ajustaron cinco funciones de volumen (Hohenald, Honer, Ogaya, Schumacher y Spurr), seleccionando aquella que presentase los mejores indicadores de ajuste.

Cada función seleccionada fue validada utilizando el procedimiento de Vallejos (1975) y de Freese (1960). Con las funciones validadas se estimó el volumen de cada parcela.

Se llevó a cabo un análisis de varianza del incremento medio anual en volumen por hectárea (IMA $\mathrm{m}^{3} / \mathrm{ha} / \mathrm{año}$ ) considerando como fuente de variación la serie de suelo que sustentaban las plantaciones y como covariable el número de árboles por hectárea, ya que este no permaneció constante. 


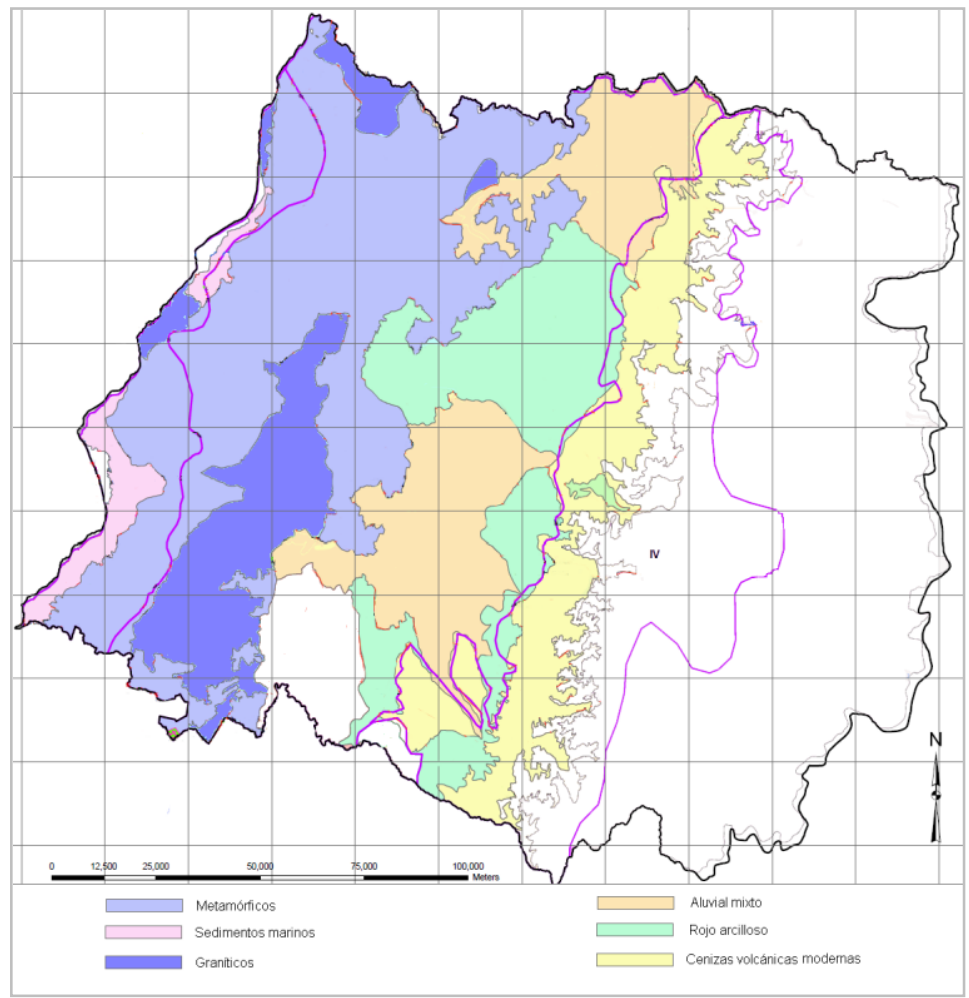

Figura $\mathrm{N}^{\circ} 1$

ZONAS DE CRECIMIENTO DE Eucalyptus globulus EN LA REGIÓN DEL MAULE Y ZONIFICACIÓN POR TIPO DE SUELO

\section{RESULTADOS Y DISCUSIÓN}

La cubicación de cada zona se hizo con la función de Schumacher $\left(V=b_{0} D A P^{b 1} h^{b 2}\right)$, que presentó un coeficiente de variación ajustado que varió entre 0,9961 y 0,9971 y un error estándar de estimación de $0,01 \mathrm{~m}^{3}$. Las funciones fueron exactas e insesgadas, pero la excepción fue la zona 2 donde se detectó sesgo variable que fue corregido aplicando la predicción inversa (Ostle, 1973 citado por Vallejos, 1979).

El IMA en volumen tuvo un amplio rango de dispersión con valores entre 1,12 y 39.96 $\mathrm{m}^{3}$ ha/año y un promedio de $9,96 \mathrm{~m} / \mathrm{ha} /$ año (Figura $\mathrm{N}^{\circ}$ 2). Como el número de árboles por hectárea condiciona el resultado del IMA en volumen (Cuadro $N^{\circ} 1$ ), se calculó el IMA en volumen de cada árbol, que resulta de dividir el IMA en volumen por el número de árboles por hectárea. Estos valores fluctuaron entre 0,0067 y $0,0235 \mathrm{~m}^{3} / \mathrm{ha} / \mathrm{año}$, con un promedio de 0,0126 , que resulta superior al reportado por INFOR-CORFO (1989), pero inferiores a los reportados por Santelices (2005) que alcanzaron los $0,0240 \mathrm{~m}^{3} / \mathrm{ha}$ año por árbol. El riego en el ensayo de Santelices (2005) favoreció la expresión volumétrica de cada árbol, práctica que es rentable si se hace por surcos (Guerra et al., 2010). 


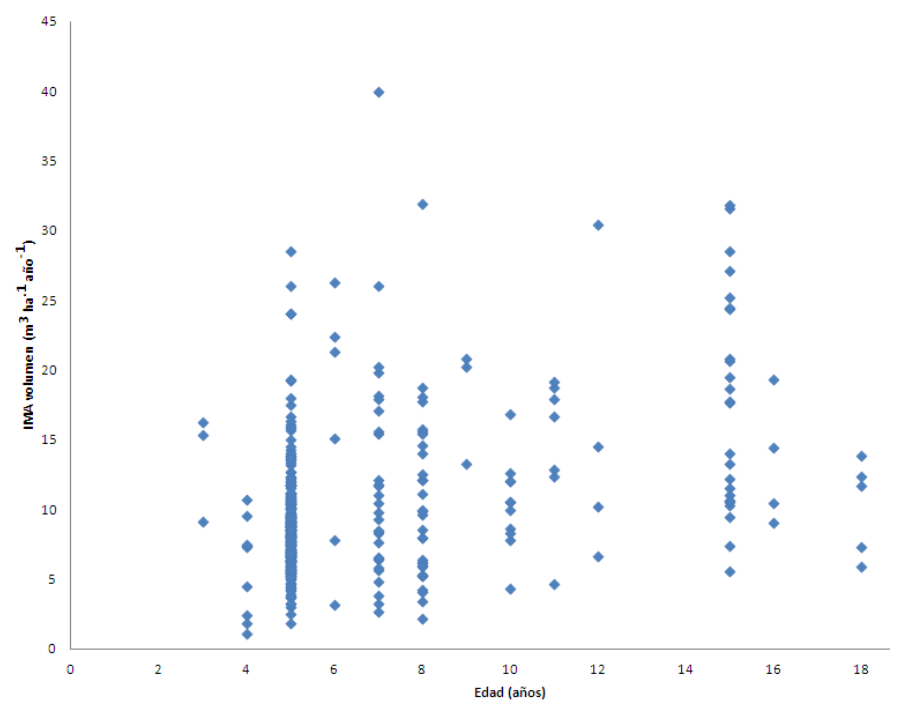

Figura $N^{\circ} 2$

DISPERSIÓN DEL IMA EN VOLUMEN EN PLANTACIONES DE Eucalyptus globulus EN LA REGIÓN DEL MAULE

El IMA en diámetro y altura (Cuadro $\mathrm{N}^{\circ} 1$ ) es muy superior a los valores reportados por INFOR-CORFO (1989) para la misma zona, ya que estos no superaban los $1,1 \mathrm{~cm}$ en diámetro y los $1,4 \mathrm{~m}$ en altura, pero similares a los obtenidos por Santelices (2005).

Cuadro $\mathrm{N}^{\circ} 1$

DESCRIPCIÓN DASOMÉTRICA DE LAS MEDICIONES Y ESTIMACIONES REALIZADAS

\begin{tabular}{|c|c|c|c|c|}
\hline \multirow[b]{2}{*}{ Tipo de Suelos } & \multicolumn{3}{|c|}{ IMA } & \multirow{2}{*}{$\begin{array}{c}\text { Promedio } \\
\text { Árboles } \\
\left(\mathbf{N}^{\circ} / \mathrm{ha}\right)\end{array}$} \\
\hline & $\begin{array}{l}\text { DAP } \\
(\mathbf{c m})\end{array}$ & $\begin{array}{c}\mathbf{h} \\
(\mathrm{m})\end{array}$ & $\begin{array}{c}\text { Volumen } \\
\left(\mathrm{m}^{3} / \mathrm{ha}\right)\end{array}$ & \\
\hline \multicolumn{5}{|c|}{ Zona de Crecimiento 1} \\
\hline Aluvial mixto & 2,1 & 2,0 & $9,4344 \pm 0,2245$ & 1.053 \\
\hline Cenizas volcánicas modernas & 2,0 & 2,1 & $13,3404 \pm 1,2636$ & 741 \\
\hline Graníticos & 1,8 & 1,7 & $6,9202 \pm 0,2348$ & 1.030 \\
\hline Metamórficos & 1,8 & 1,9 & $11,7367 \pm 1,1025$ & 969 \\
\hline Rojo arcilloso & 1,4 & 1,8 & $17,0991 \pm 1,2155$ & 1.054 \\
\hline Sedimentos marinos & 1,6 & 1,7 & $8,3575 \pm 1,7497$ & 721 \\
\hline \multicolumn{5}{|c|}{ Zona de Crecimiento 2} \\
\hline Graníticos & 1,4 & 1,9 & $13,0722 \pm 2,1570$ & 1.331 \\
\hline Metamórficos & 1,4 & 1,8 & $8,9582 \pm 2,1152$ & 1.318 \\
\hline Sedimentos marinos & 1,7 & 2,0 & $13,2828 \pm 4,7458$ & 1.056 \\
\hline \multicolumn{5}{|c|}{ Zona de Crecimiento 4} \\
\hline Cenizas volcánicas modernas & 2,2 & 2,9 & $20,5557 \pm 3,0708$ & 875 \\
\hline
\end{tabular}


No fue posible realizar un análisis de varianza multifactorial incorporando como fuentes de variación las zonas de crecimiento y los tipos de suelos, ya que los tipos de suelos no eran similares en las zonas.

El análisis paramétrico por zona de crecimiento y por tipo de suelo (Cuadro $\mathrm{N}^{\circ} 2$ ) indicó la existencia de diferencias significativas. No se cumplieron los supuestos de este análisis, de modo que se llevó a cabo la prueba de Kruskal-Wallis, que ratificó las diferencias entre las fuentes de variación.

\section{Cuadro $\mathrm{N}^{\circ} 2$}

ANÁLISIS DE VARIANZA PARAMÉTRICO Y NO PARAMÉTRICO

\begin{tabular}{|l|l|r|r|r|r|}
\hline \multirow{2}{*}{ Tipo de Análisis } & \multirow{2}{*}{ Fuente Variación } & \multicolumn{2}{|c|}{ Análisis Paramétrico } & \multicolumn{2}{|c|}{ Análisis no Paramétrico } \\
\cline { 3 - 6 } & & Valor F & \multicolumn{1}{c|}{$\mathbf{p}$} & Kruskal-Wallis & p \\
\hline Zona & N $^{\circ}$ árboles $\left.{ }^{*}\right)$ & 39.17 & 0.000 & & \\
Crecimiento & Zona Crecimiento $^{*}$ & 8.04 & 0.000 & 12.09 & 0.002 \\
\hline \multirow{2}{*}{ Tipo de Suelo } & N $^{\circ}$ de árboles( $\left.{ }^{*}\right)$ & 69.84 & 0.000 & & \\
& Tipo de Suelo & 33.77 & 0.000 & 121.07 & 0.000 \\
\hline
\end{tabular}

$\left(^{\star}\right)$ El número de árboles por hectárea fue la covariable, que resultó ser altamente significativa.

p: Probabilidad

Las zonas de crecimiento presentaron dos grupos homogéneos (Cuadro $\mathrm{N}^{\circ} 3$ ), el primero conformado por la zona 1 y 2 y el segundo por la zona 4. Queda de manifiesto que la zona precordillerana presenta mayores incrementos en volumen que los registrados en la depresión intermedia y zona costera de la Región del Maule. Los tipos de suelos están representados en tres grupos, el primero compuesto por los suelos Graníticos que presentaron el menor incremento volumétrico. El grupo intermedio está compuesto por los suelos Aluviales Mixtos, Metamórficos y Sedimentos Marinos. El grupo con mayor incremento volumétrico está conformado por las Cenizas Volcánicas y los Rojo arcillosos.

\section{Cuadro $\mathrm{N}^{\circ} 3$}

PRUEBA DE COMPARACIÓN MÚLTIPLE STUDENT NEWMAN KEULS (SNK)

\begin{tabular}{|c|c|c|c|c|c|}
\hline \multirow{2}{*}{\multicolumn{2}{|c|}{ Fuente de Variación }} & \multicolumn{2}{|c|}{ Análisis Paramétrico } & \multicolumn{2}{|c|}{ Análisis no Paramétrico } \\
\hline & & \multirow{4}{*}{$\begin{array}{r}\text { Promedio } \\
9,8650 \\
10,0260 \\
21,4263\end{array}$} & $\begin{array}{c}\text { Grupos } \\
\text { Homogéneos }\end{array}$ & \multirow{2}{*}{$\begin{array}{r}\text { Rangos } \\
216,0\end{array}$} & $\begin{array}{c}\text { Grupos } \\
\text { Homogéneos }\end{array}$ \\
\hline \multirow{3}{*}{ Zona Crecimiento } & Zona 1 & & $a$ & & $a$ \\
\hline & Zona 2 & & $a$ & 232,2 & a \\
\hline & Zona 4 & & $b$ & 407,3 & $b$ \\
\hline \multirow{6}{*}{ Tipo Suelo } & Graníticos & 7,5967 & $a$ & 148,6 & $a$ \\
\hline & Aluvial Mixto & 9,3451 & $\mathrm{~b}$ & 224,4 & $\mathrm{~b}$ \\
\hline & Metamórficos & 10,6703 & $\mathrm{~b}$ & 239,9 & $b$ \\
\hline & Sedimentos Marinos & 10,7772 & $b$ & 260,3 & $b$ \\
\hline & Cenizas Volcánicas & 16,4646 & c & 340,7 & $c$ \\
\hline & Rojo Arcilloso & 16,9986 & c & 355,3 & $c$ \\
\hline
\end{tabular}


Las estimaciones de $25 \mathrm{~m}^{3} / \mathrm{ha} /$ año de CONAF (2012) en suelos Metamórficos de la Región del Maule con silvicultura de precisión, superan las estimaciones de este estudio.

\section{CONCLUSIONES}

Se presentan diferencias estadísticas en la productividad de las plantaciones de Eucalyptus globulus en la Región del Maule.

Las plantaciones establecidas en la precordillera en Cenizas volcánicas modernas y suelos Rojo arcilloso presentan el mayor incremento medio anual en volumen.

La depresión intermedia y la zona costera con suelos Aluvial Mixto, Metamórficos y Sedimentos Marinos presenta incrementos intermedios.

Los suelos graníticos presentan el menor incremento volumétrico.

\section{REFERENCIAS}

CONAF, 2012. Plantaciones Dendroenergéticas de Eucalyptus globulus: Un Impulso al Desarrollo. Disponible en: http://www.conaf.cl/bosques/noticia-plantaciones_dendroenergeticas_deeucalyptusglobulus_un_impulso_al_desarrollo-1461.html

Freese, F., 1960. Testing Accuracy. Forest Science. 6 (2): 139 - 145

Guerra, E.; Herrera, M. y Drake, F., 2010. Efecto de los sistemas de riego en la rentabilidad de plantaciones de eucalipto (Eucalyptus globulus). Agrociencia [online]. 2010, vol.44, n.1 [citado 2012-10-26], pp. 99-107 . Disponíblel en: <http://www.scielo.org.mx/scielo.php?script=sci_arttext \&pid=S1405-31952010000100010\&lng=pt\&nm=iso>. ISSN 1405-3195.

INFOR-CORFO, 1989. Eucalyptus Principios de Silvicultura y Manejo. Ed. Prado. J.; Barros. S. Instituto Forestal Chile - Corporación de Fomento de la Producción. 199 p.

INE, s/f. Cuadro 17: Superficie de las Plantaciones Forestales por Especie, en las Explotaciones Forestales, según Región, Provincia y Comuna. Instituto Nacional de Estadísticas Chile.

Disponible en: http://www.ine.cl/canales/chile_estadistico/censos_agropecuarios/censoagropecuario_07_comunas.php

Pampolina, N. M., Dell, B., Malajczuk, N., 2002. Dynamics of ectomycorrhizal fungi in an Eucalyptus globulus plantation: effect of phosphorus fertilization. Forest Ecology and Management. 2002, vol. 158 № 1-3, p. 291-304.

Santelices. R., 2005. Desarrollo de una plantación de Eucalyptus globulus establecida en primavera con diferentes tratamientos de Riego. Bosque 26(3): 105-112. 2005.

Vallejos. C. 1979. Un método para evaluar la exactitud de la función de volumen. Instituto Forestal. Santiago Chile. Informe técnico no 75.23 p. 
